\title{
Ser mujer hipertensa: una experiencia silenciosa en la familia y en la sociedad
}

\section{Hypertensive women: a silent experience in family and society}

\section{Ser Mulher Hipertensa: uma experiência silenciosa na família e na sociedade \\ Mónica Margarita Barón Castro ${ }^{1}$, Mercy Soto Chaquir ${ }^{2}$.}

${ }^{1}$ Profesora Titular, Programa de Enfermería, Facultad de Ciencias de la Salud, Fundación Universitaria del Área Andina, Pereira, Colombia.e-mail: momabaca35@hotmail.com

${ }^{2}$ Coordinadora Académica, Programa de Enfermería, Facultad de Ciencias de la salud, Universidad Libre, Pereira, Colombia. e-mail:mercysotochaquir@hotmail.com

Cómo citar este artículo en edición digital: Barón Castro, M.M; Soto Chaquir, M. (2012) Ser Mujer Hipertensa: una experiencia silenciosa en la familia y en la sociedad.16, 34. Disponible en: <http://dx.doi.org/10.7184/cuid.2012.34.04> Correspondencia: Correspondencia: Mónica Margarita Barón Castro. Facultad de Ciencias de la Salud. Fundación Universitaria del Área Andina, Pereira, Colombia.e-mail: momabaca35@hotmail.com.

Recibido 12/03/2012/ Aceptado: 10/06/2012

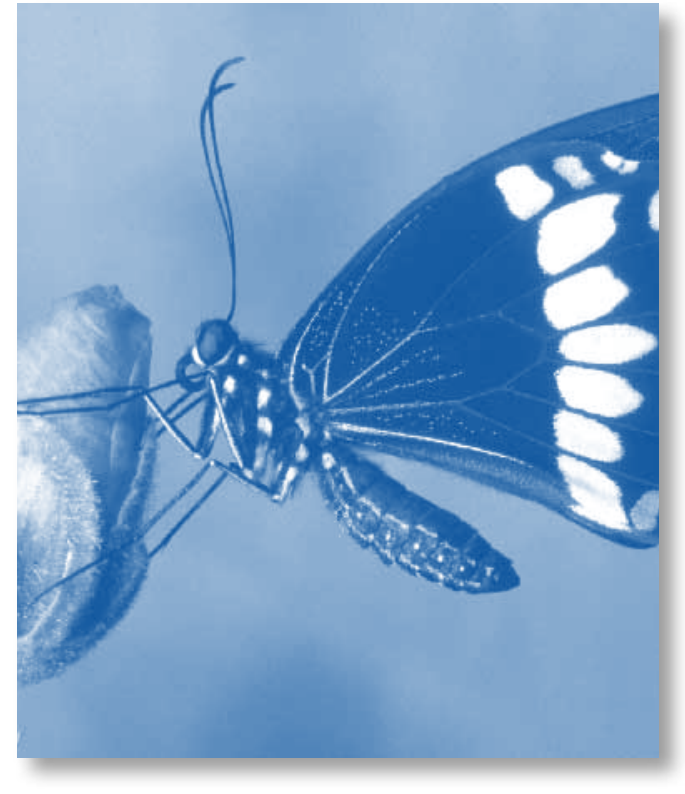

ABSTRACT

Introduction: Hypertension has implications for public health indicators and quality of life of people and taking as a principle of autonomy of adults in making decisions against their health, this research seeks to understand the meaning that a group of hypertensive elderly women gave hypertension and their social environment.
Methodology: This is a phenomenological study in the perspective of Merleau Ponti. Six women were interviewed who was volunteered to participate signed the informed consent record. Each one was conducted at home, in three depth - interviews.

Results: Two thematic areas were identified for understanding the phenomenon, meaning "living with a silent disease but serious consequences" and "social environment: coadjuvant or limiting health care." Hypertension was living with anxiety and concern. The social environment was a horizon of uncertainty, indifference, family, disrespect and abuse, and overload voltage generator, offset in part by support from neighbors and friends. Physical disability, coupled with long waits, alienated women from health institutions. We identified a lack of clarity on the continuing needs for monitoring and control of health.

Conclusions: Social environment and the meaning given the same involved in the lack of adherence to treatment as well as the silent disease, meaning that as the participants gave to it. The connotation of women as caregivers, 
the social role ascribed and socially acceptable representations of what is a woman represented a very important role in the low self of the participants.

Recommendations: Transcending the attention of healing attention towards health that provided the specific conditions and social environment is needed to improve health care of people. Develop programs hypertension with home care programs, emerges as an important alternative nursing care to these people.

Keywords: Elderly, Women's health, hypertension, nursing, phenomenology, lifechanging events, quality life.

\section{RESUMO}

Introdução: A hipertensão arterial tem consequências nos indicadores de saúde pública e na qualidade de vida das pessoas, tendo como principio a autonomia dos adultos na tomada de decisões com sua saúde. Esta pesquisa pretende compreender a relação da hipertensão e o meio social de um grupo de mulheres hipertensas já com a idade avançada.

Métodos: Estudo fenomenológico na perspectiva de Merleau Ponti, sendo entrevistadas seis mulheres que aceitaram voluntariamente participar, assinando um termo de consentimento. Com cada uma delas foram realizadas três entrevistas com profundidade no domicílio delas.

Resultados: Foram identificadas 2(duas) temáticas que permitiram compreender o fenômeno: significado de "viver com uma doença silenciosa mas de graves consequências" e "meio social: coadjuvante ou limitadora para o cuidado da saúde". A hipertensão foi vivida com angustia e preocupação. O meio social mostrou um horizonte de incerteza, indiferença familiar, desconsideração e abuso, gerador de tensão e sobrecarga, compensado em parte pelo apoio de vizinhos e amigos. A incapacidade física, associada a longas esperas, alijaram as mulheres das instituições de saúde. Identificou-se a falta de instrução sobre a necessidade permanente do seguimento e controle da saúde.

Conclusões: $\mathrm{O}$ meio social e o significado dado a ele intervieram na falta de adesão ao tratamento bem como na relação de como a doença foi adquirida de forma silenciosa pelas participantes. A conotação social da mulher como cuidadora, o rol social atribuído e as representações socialmente aceitas do que é ser mulher; representaram um papel muito importante no baixo autocuidado das participantes.

Recomendações: Transcender a atenção de curar a uma atenção em prol da saúde em que contemple as condições particulares e o meio social é necessário para melhorar o cuidado da saúde das pessoas. Desenvolver os programas de hipertensão arterial com programas de cuidado em casa podem alternativas importantes no cuidado que a enfermagem possa proporcionar a estas pessoas.

Palavras-chave: adulto mais velho, saúde da mulher, hipertensão arterial, enfermagem, fenomenologia, acontecimentos que mudam a vida, qualidade de vida.

\section{RESUMEN}

Introducción: La hipertensión arterial tiene consecuencias en los indicadores de salud pública y en la calidad de vida de las personas, y teniendo como principio la autonomía de los adultos en la toma de decisiones frente a su salud, esta investigación busca comprender el significado que un grupo de mujeres adultas mayores hipertensas le dio a la hipertensión y a su medio social. 
Métodos: Estudio fenomenológico en la perspectiva de Merleau Ponti. Se entrevistaron seis mujeres que aceptaron voluntariamente participar firmando acta de consentimiento informado. A cada una se le realizaron tres entrevistas a profundidad en el domicilio.

Resultados: Se identificaron 2 áreas temáticas que permitieron comprender el fenómeno: significado de "vivir con una enfermedad silenciosa pero de graves consecuencias" y "medio social: coadyuvante o limitante para el cuidado de la salud". La hipertensión fue vivida con angustia y preocupación. El medio social mostró un horizonte de incertidumbre, indiferencia familiar, desconsideración y abuso, generador de tensión y sobrecarga, compensado en parte por el apoyo de vecinos y amigos. La incapacidad física, aunada a largas esperas, alejaron a las mujeres de las instituciones de salud. Se identificó falta de claridad en la necesidad permanente del seguimiento y control de la salud.

Conclusiones: El medio social y el significado dado al mismo intervinieron en la falta de adherencia al tratamiento así como el significado que como enfermedad silenciosa dieron las participantes a la misma. La connotación social de la mujer como cuidadora, el rol social atribuido y las representaciones socialmente aceptadas de lo que es ser mujer; representaron un papel muy importante en el bajo autocuidado de las participantes.

Recomendaciones: Trascender la atención del curar a una atención en pro de la salud en la que se contemple las condiciones particulares y del medio social es necesario para mejorar el cuidado de la salud de las personas. Desarrollar los programas de hipertensión arterial con programas de cuidado en casa, surge como una alternativa importante en el cuidado de enfermería a estas personas.
Palabras clave: adulto Mayor, salud de la Mujer, hipertensión arterial, enfermería, fenomenología, acontecimientos que cambian la vida.

\section{INTRODUCCIÓN}

El incremento de las enfermedades crónicas no transmisibles afecta a la población mundial en forma alarmante (Chobanian, et al. 2003); la hipertensión arterial señala la necesidad de nuevas y mejores intervenciones que conduzcan a promover y conservar la salud de las personas (Colombia. Ministerio de la Protección Social, 2000). La Organización Mundial de la Salud (OMS) para el año 2003, identificó 700 millones de personas en todo el mundo con cifras tensionales altas, o sea, alrededor del $15 \%$ de la población mundial diagnosticada como hipertensa, cerca del $30 \%$ de la misma padece esta enfermedad sin saberlo (Rodríguez y Marín, 2007). Para el año 2005, la misma OMS manifestó que tres factores de riesgo de enfermedades crónicas: el consumo de tabaco, la hipertensión arterial y la hipercolesterolemia, figuran entre las diez causas principales de enfermedad en todas las regiones (OMS, 2005).

La hipertensión arterial afecta principalmente a personas mayores de 45 años y el riesgo se incrementa con la edad, en la mujer mayor de 50 años supera la prevalencia respecto a los hombres (Journal of the American College of Cardiology, 2006). En el 2008, para Colombia, la prevalencia de la hipertensión arterial en la población mayor de 15 años fue de 12,6\% (Serpa, 2008).

Entre las barreras más importantes para la prevención y la detección oportuna de la hipertensión arterial están el desconocimiento de los factores de riesgo y su curso asintomáti- 
co inicial. En Argentina alrededor del 50\% de las mujeres de la tercera edad que tuvieron alta prevalencia de factores de riesgo cardiovascular, los desconocían, evidenciando una falla en el área de prevención cardiovascular en los sistemas de salud (Nigro, 2005). Se calcula que unos 20 millones de personas que tienen hipertensión arterial en los Estados Unidos son ajenas a la peligrosa disfunción que sufren (Chobanian, 2003).

Una vez diagnosticada la hipertensión arterial, el cuidado se convierte en el aspecto más importante para evitar las complicaciones. Entre las medidas de cuidado se destacan: prescripción farmacológica, asistencia a controles, recomendaciones impartidas por el equipo de salud respecto a modificaciones en el estilo de vida tales como una rutina de ejercicios físicos, control de riesgo de sobrepeso, control del estrés y modificación de hábitos alimenticios (Rosano, Vitale, , Tulli, 2007 ), cuyo incumplimiento genera alto riesgo de complicaciones cardio-cerebro-pulmonares y renales, deteriora la calidad de vida de forma integral y lleva incluso a la muerte. (National Heart Lung and blood Institute), (Braunwald y Harrison, 2005), (Colunga, 2008).

Los estudios citados focalizan la atención en el conocimiento de la experiencia vivida por mujeres adultas hipertensas, para lo cual es necesario explorar la connotación cultural de género. La cultura se entiende como la red o trama de sentidos con que se da significado a los fenómenos o eventos de la vida cotidiana (Geertz, 1987). En Latinoamérica, la identidad de género asignada a las mujer tiene que ver con el deber ser. A ella se le dice cómo tiene que actuar, ser y pensar. Estas órdenes se cumplen a través de un cuerpo, que está en el centro de las definiciones sociales. En este proceso de reproducir la función social del género, las

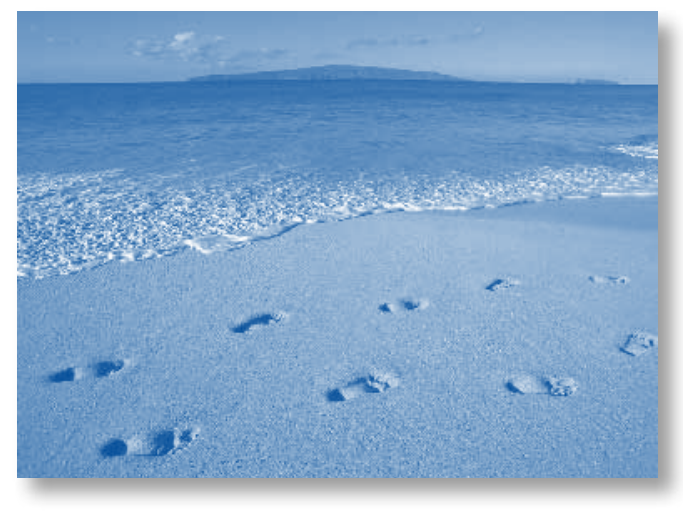

mujeres aprenden a ser madres, y este ser tiene sus raíces en la otredad. En este "ser para otros" las mujeres encuentran su realización, pues a través de la cultura más que mujeres son llamadas a ser madres. Tiene además que ver con la autoidentidad, que es la forma en que ellas se miran y se nombran, ya que ser madre significa ser buena y ser santa (Lagarde, 2004).

El interactuar con la mujer adulta hipertensa y acercarse a su experiencia de salud es identificarse con una forma de conocer emociones, temores, imaginarios, visiones de mundo; es retomar la salud como un valor, desde el sentir propio y la experiencia particular, y no asumirlo únicamente como un referente social de bienestar individual o colectivo.

En la cultura latinoamericana, hablar con propiedad de la salud de las mujeres es también hablar del cuerpo de las mujeres, un cuerpo con tabúes, simbolizado y significado con valores que las alejan de él, un cuerpo ajeno al autoconocimiento, que encierra una vida plena de vitalidad, de fortaleza interior, de preocupaciones y ocupaciones (Palma, 2001). Esta relación con el cuerpo tiene que ver directamente con el cómo las mujeres se ubican en sus espacios inmediatos de interacción social, con una historia particular, con la vivencia de su ser y su enfermedad, cargando con una herencia cultural e histórica. La maternidad forma parte del conocimiento tradicional, con 
respecto al cómo debe pensar una mujer, cómo deber actuar y cuáles deben ser sus preocupaciones y ocupaciones (Basaglia y Kanoussi, 1985).

Bajo estas consideraciones, la mujer no crea una conciencia de vida, de cuerpo, de trabajo y de cuidado para sí misma, sino para su aplicación con el otro y se aprecia y califica en función de su capacidad de atender a los demás (Guilligan, 1985). Por 1o tanto, la mujer no acepta ni comprende la salud como un valor al que tienen derecho, no existe en ella un referente de autocuidado culturalmente válido.

Ante las implicaciones de la hipertensión y sus consecuencias en los indicadores de salud pública y en la calidad de vida, y teniendo como principio la autonomía del ser humano en la toma de decisiones frente a su salud, se buscó comprender el significado que un grupo de mujeres adultas mayores hipertensas le dio a la hipertensión y a su medio social.

\section{METODOLOGÍA}

Estudio cualitativo abordado con fenomenología interpretativa, en la perspectiva de Merleau Ponti (2008). Las participantes cumplieron como criterios de inclusión: sexo femenino, mayores de 50 años, diagnóstico médico de hipertensión arterial, poseer condiciones físicas y mentales para responder a las entrevistas, aceptar participar voluntariamente $y$ firmar el consentimiento informado.

Las participantes fueron contactadas telefónicamente, se les solicitó autorización para ser visitadas en su domicilio, donde se explicó el objetivo del estudio.

Después de realizadas las entrevistas fueron grabadas en audio casetes y transcritas textualmente por las investigadoras, adicionalmente, se analizaron notas observacionales, metodológicas y analíticas registradas por las investigadoras durante las entrevistas (Carvalho, 1991).

Se realizó muestreo teórico, la saturación de los datos permitió la participación de seis mujeres, a cada una se le realizaron tres entrevistas en profundidad. Las preguntas orientadoras fueron: ¿Qué significa para Usted ser hipertensa? y ¿cómo percibe el medio social frente a su situación de salud?

El análisis de los datos se hizo siguiendo los postulados de la fenomenología interpretativa descritos por Patricia Benner; para lograr la comprensión de las narraciones se exige la lectura rigurosa del texto (transcripción de las entrevistas en profundidad), lo cual a su vez incluye preguntar, comparar y vivir imaginariamente en las situaciones; este proceso involucra el análisis, la síntesis, el criticismo y la comprensión, como habilidades que permiten articular el significado del texto y generar comentarios interpretativos (Benner Associates. 1991). En el rigor metodológico se tuvo en cuenta los criterios de coherencia, credibilidad, aplicabilidad, transferibilidad y auditabilidad, descritos por Denisse Polit (2000).

\section{RESULTADOS}

Participaron seis mujeres de edades entre 55 y 70 años; cinco eran madres cabezas de hogar sin pareja y una vivía con su pareja. En promedio tenían 3 hijos y todas convivían con familiares. Gran parte de los hijos eran mayores de edad que habían formado sus propios hogares y acompañaban a sus madres de manera temporal. Todas procedían de estrato socioeconómico bajo, tenían nivel primario de escolaridad. Sólo dos participantes tenían vivienda propia; las demás, pagaban arriendo o vivían en casa de sus hijos de quienes dependían económicamente. 
De las voces de las entrevistadas surgieron dos grandes temas: el significado de vivir con una enfermedad silenciosa pero de graves consecuencias y el medio social como coadyuvante o limitante para el cuidado de la salud.

El significado de vivir como hipertensas se enmarcó en un continuum de lo invisible a lo visible, las mujeres se enfrentaron a un enemigo que inicialmente no se manifestó, no les causó limitaciones para las actividades de la vida diaria, ni deterioró su capacidad de desempeño; esto les permitió seguir viviendo sin reconocer la enfermedad, hasta el momento en que se manifestaron las complicaciones, hecho que les condujo al reconocimiento de la hipertensión arterial y a experimentarla como "el enemigo silencioso que come por dentro", pues generó pérdidas afectivas, sociales y físicas, que significaron dejar de ser lo que había sido alguna vez. Las entrevistadas así lo expresaron:

- “...como nada me dolía, de vez en cuando me dolía la cabeza y me tomaba un ibuprofeno y ya se me pasaba... "...La verdad, durante todo este tiempo no asistí a los controles ni tome pastas porque como no sentía nada, no me dolía nada...no volví al médico porque como nada sentía"

- "... Como esa es una enfermedad silenciosa, es callada, esta come por dentro, esa no brota, brota cuando ya está pa' morirse uno... porque como yo, vea, le dan borracheras, se está cayendo, y vea que se cayó y se cayó de verdad"

- “.. esta enfermedad me cambió la vida”

El medio social como limitante o coadyuvante para el cuidado de la salud se expresó desde dos perspectivas: la familia y las instituciones de salud. En la dinámica familiar, su rol social como mujer se antepuso al cuidado de su salud, predominó su compromiso de cuidadora, que unido a las condiciones de deterioro progresivo de la salud, les generó tensión y sobrecarga manifestada en inasistencia a controles médicos, abandono del tratamiento, aislamiento social; en forma compensatoria, algunas mujeres encontraron refugio en sus vecinos y amigos que constituyeron un alivio en la dificultad. Las instituciones de salud fueron percibidas como contextos que dificultaron o limitaron un oportuno y adecuado autocuidado, entre amabilidad, largas esperas y desinformación.

El estar enferma, le exigió cuidados especiales, cambios en la rutina, asistencia a controles y medicación, nuevas formas de interactuar con su medio social y de percibir su realidad. Con el paso de los años, las mujeres se enfrentaron a cambios en su rol social y familiar; su rol se invirtió: de cuidadoras pasaron a ser receptoras del cuidado, la transición a la dependencia condujo a experimentar agotamiento de energía, que les generó conflicto, tristeza y culpa, como fue expresado:

“...me siento muy incómoda, pues yo estoy muy bien aquí donde mi hija, pero hay veces que me da como pena de estarle pidiendo a la hija porque uno ya tan enfermo...pues nadie le da trabajo y mi hija también tiene sus obligaciones"

En algunos casos, su situación les permitió sentirse útiles y continuar como eje central de la función familiar pero en otros, les generó tensión y sobrecarga:

“...yo dejo de ir a los controles, de salir con la vecina a caminar, porque...es que es más importante los deberes de la casa que hay que lavar, planchar, que la casa esté presentable $y$ cuidar a mi nieto."

Los vecinos jugaron un papel significativo, el apoyo social que recibieron de ellos condujo a la mujer adulta mayor en su condición de hipertensa, a sentir que eran amadas, queridas, importantes en la vida de ellos. 
“...gracias a Dios tengo a mi vecina que me conoce ya varios años y ella es la que me ha dado la mano cuando la necesito"

Los lazos con sus vecinas se caracterizaron por ser fuertes y frecuentes, adquirieron un sentido de acompañamiento, ayuda y de seguridad. La frecuencia con la cual pudieron recibir ese apoyo social, se hizo posible por la cercanía física, por el mayor tiempo que la mujer hipertensa permanece en el hogar y por darse la condición de vecindad por muchos años.

Por su parte, la relación con los servicios de salud se mostró como una limitante para el cuidado de su salud. El madrugar para solicitar la cita fue considerado como preocupante, y muy peligroso, por las condiciones de inseguridad alrededor de sus residencias, situación que ponía en riesgo su integridad física y, así fue expresado:

“.. pero vea que problema, las citas siempre las dan para por la mañana y uno tiene que madrugar mucho y todo este barrio (sector de la Ciudad) es muy peligroso...entonces nooo yo mejor me quedo en la casa"

Adicionalmente, la incomodidad generada por los prolongados tiempos de espera fue otro factor que dificultó la asistencia de las participantes a sus controles regulares. Los trámites administrativos, largas filas y demora para la atención les significaron interferencias en su actividad cotidiana, incumplimiento de compromisos laborales, descuido de la familia. La necesidad de atender a su familia, junto con la de su propio autocuidado por la hipertensión, desencadenó conflicto a las participantes, quienes la mayor parte de las veces optaron por atender los asuntos diferentes a su salud.

“...Allá es muy demorado y aquí me necesitan sea por la mañana o sea al medio día, o sea tendría que ser que no me demoraran tanto porque toca volver a hacer la comida, porque se venden almuerzos, desayunos y comidas ... o sea, si me voy a las siete de la mañana a las nueve o diez de la mañana tengo que estar aquí y allá no me despachan ligero, y ya a las doce no me alcanza el tiempo para estar aquí... yo tenía mis controles muy al orden del día pero no he vuelto porque es muy aburrido eso de que lo mandan a uno a facturar allí y le toca a uno hacer una cola de veinte metros y llega uno a la ventanilla y que falta un papel y otra vez a hacer la cola por otros veinte metros o si no es que le dicen a uno siéntese allí y es uno espere que espere y nada entonces yo mejor me voy"

A manera de síntesis, las mujeres adultas mayores con hipertensión arterial, entrevistadas para este estudio mostraron una vivencia que las coloca en situación de alta vulnerabilidad en varios sentidos: personal, emocional, cultural, económica y social; vivían con una enfermedad crónica, en algunos casos incapacitante, con una connotación cultural de "ser mujer" con el estigma de cuidadora, preocupadas por no cumplir plenamente con el rol social asignado, con sentido de menoscabo de su vitalidad, un bajo poder adquisitivo, baja escolaridad y dificultades para relacionarse con el medio. En sus quejas, las mujeres evaluaron su propia salud como mala, de manera reiterada se refirieron a la pérdida de la memoria y sus repercusiones en sus actividades cotidianas y la profunda tristeza que sienten en su cotidiano vivir. Aún así, algunas mujeres se mostraron resignadas y con deseos de continuar su vida, pero siempre esforzándose por satisfacer las necesidades de los otros, que se anteponían al cuidado de su propia salud.

\section{DISCUSIÓN}

$\mathrm{Al}$ expresar la vivencia de la hipertensión arterial, las mujeres adultas orientan su discurso hacia dos momentos concretos que re- 
velan la trascendencia que tiene en el cuidado de la salud al reconocer y experimentar la presencia de la enfermedad. De la ausencia de síntomas, una característica clínica del inicio de la hipertensión arterial que la ha llevado a ser denominada por varios autores como enfermedad silenciosa (Iza- Stoll, 2006), pasa a serias manifestaciones clínicas, crisis hipertensivas y complicaciones inesperadas, que expresan la gravedad del problema. Como lo señala la literatura médica, la hipertensión, en sus inicios silenciosa, es una alteración lentamente progresiva que con el paso de 10 a 20 años ocasiona daños significativos en órganos blancos (Colombia. Ministerio de la Protección Social).

El menoscabo de la capacidad física, laboral y recreativa, como consecuencia de la hipertensión arterial y sus complicaciones, contribuye a que las mujeres adultas perciban con claridad sus pérdidas. Su salud ya no es igual, señalan deterioro de sus funciones orgánicas, cambios drásticos en su estilo de vida, en lo laboral, social y emocional; sienten aminorada su energía vital y su calidad de vida. Rengifo y Henríquez (2010) encuentran una relación estadísticamente significativa entre la experiencia de pérdidas físicas y la calidad de vida del adulto mayor, con lo que estos autores denominan la salud social; a su vez, identifican que con la ocurrencia de las pérdidas sociales hay también una disminución en la salud física.

En la situación de la mujer adulta que padece hipertensión arterial confluyen dos fenómenos simbióticos que se expresan en carencias que permiten configurar el significado de la enfermedad como un dejar de ser lo que había sido alguna vez. El ser adulta mayor, acorde con lo expresado por Erickson (Bordignon, 2005), coloca a la mujer en un momento inarmónico de su existencia; de forma progresiva se debilitan sus virtudes como fuerzas sincrónicas, al tiempo que se agudiza el descontento como fuerza distónica. Por otro lado, la vivencia de la enfermedad inclina en forma negativa la balanza, de un "yo" que se cuida y se cela a un "yo" que se rechaza y, desde la posibilidad de sobrevivencia como una manifestación de fuerza básica, a un menosprecio por sí misma, al ver menguada su capacidad vital.

La estructura económica, política y social ha definido por años el rol de la mujer en la sociedad y en la tradición cultural se ha reconocido a la mujer como cuidadora por antonomasia. El cuidado como actividad informal es realizado por las mujeres desde épocas milenarias, la primacía de las mujeres en el cuidado es evidente y llega a ser una característica que define su identidad; en su rol de cuidadora la mujer toma la responsabilidad de suplir las necesidades de esposo, hijos, adultos mayores, discapacitados y enfermos (Canaval, 2010). Esta es una realidad ampliamente evidenciada en este estudio.

El medio social se percibe como un elemento de apoyo o una limitante para el cuidado de la salud. Las mujeres sienten disminuida su capacidad funcional y productiva, lo que las lleva a incrementar su situación de dependencia; ya no tienen el reconocimiento de otras épocas, se descubren frágiles, limitadas, incapaces e inútiles. Enfrentar el envejecimiento unido a la hipertensión, les reduce así su calidad de vida. Palma Campos (2001) en su estudio sobre la vivencia de la salud, y el autocuidado realizado con mujeres con diabetes mellitus e hipertensión concluye que las acciones de las mujeres con respecto a su salud son un fiel reflejo de la construcción social y cultural de su identidad. Tal como se evidenció en las entrevistas aquí analizadas, el espacio a través del cual se gesta la cultura es 


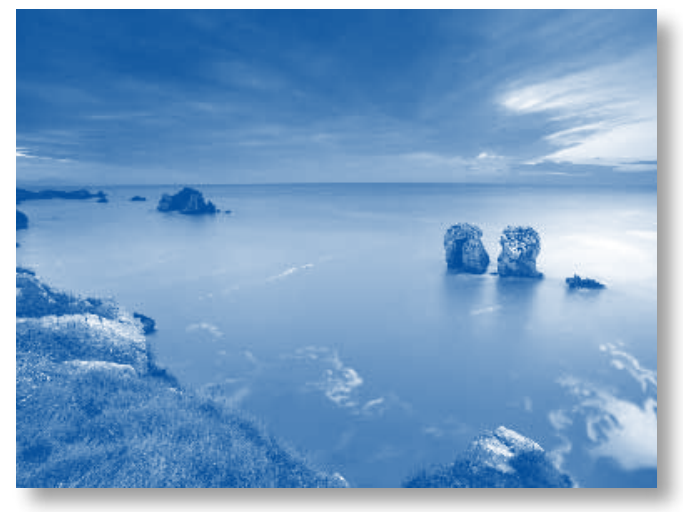

el cuerpo: un cuerpo femenino que se especializa para dedicar tiempo, cuidado y protección a las personas que pertenecen a su entorno, un cuerpo que sufre cambios que deterioran su bienestar, y en el proceso de acomodo de vivir con una enfermedad para toda la vida surge nueva autoconceptualización: se ubican como mujeres que ya no valen, que ya no sirven pues no pueden trabajar, e inclusive, que ya no tienen derechos, al no poder realizar actividades que se les imposibilita por el padecimiento.

La situación de enfermedad crónica se relaciona directamente con su capacidad de autocuidado, esta forma de aprender a estar en el mundo le impide generar cambios sustantivos dirigidos al cuidado de su propia salud. El espacio de autocuidado no es concebido por las mujeres; ellas en su cotidianidad no son capaces de cuidarse a sí mismas. Su nueva ubicación en el espacio familiar las coloca como madres enfermas, pero con las mismas obligaciones-roles a los que están acostumbrados. Este conflicto de ser tildada como buena o mala madre, al verse imposibilitada de cumplir sus funciones, le genera estrés y angustia.

La comprensión del fenómeno que envuelve a las mujeres adultas, inmersas en su medio social, que por diferentes circunstancias, incumplen las medidas de cuidado relacionadas con la hipertensión, son motivo de interés para la ciencia del cuidado. Enfermería como pro- fesión ocupa un liderazgo dentro del equipo de salud, esto facilita proyectar propuestas de promoción de la salud en todos los niveles de atención que mejoren la calidad de la asistencia brindada, la educación, la administración y la gestión de programas y planes de cuidado acordes a la realidad expresada por las personas que sufren (Durán, 2002). Se invita a entender este ser humano como un todo, que hace parte de un contexto donde permanentemente hay trasformación de una realidad compartida, que afecta a favor o en contra el cuidado de la salud

La visión holística del cuidado requiere de un espacio de interacción subjetiva, donde la mujer adulta hipertensa reciba un cuidado comprensivo, en el que a través de la experiencia del diálogo entre Enfermera- paciente se permita la expresión de la vivencia y se facilite la comprensión de los significados que las personas asignan a sus experiencias de salud. En estos significados, como lo expresa Lenninger en su teoría del cuidado transcultural (Leininger y McFarland, 2002), los valores, creencias y patrones culturales tienen una importancia trascendental cuando se procura fomentar comportamientos y estilos de vida saludables.

Aproximarse a la realidad que vive la mujer hipertensa requiere la aplicación de un cuidado humanizado. Jean Watson (Tomey, 2000) plantea este cuidado en una terapéutica básica relacional, trans-personal e intersubjetiva cimentada en la legitimidad y la sinceridad de la enfermera o enfermero que escucha $y$ brinda el cuidado con autenticidad, como una relación de ayuda y confianza, promoviendo y aceptando la expresión de los sentimientos positivos y negativos de la realidad que acompaña la vivencia de la mujer hipertensa, con el propósito de infundirle fe y esperanza en medio de las dificultades. 
La enfermera debe promover el autocuidado basado en la enseñanza y aprendizaje interpersonal y el uso del método de la resolución de problemas para la toma de decisiones acordes a las expectativas y necesidades que las mujeres hipertensas expresan, de tal manera que logren encontrar dentro de sí mismas la riqueza de la paz y la conciliación con su cuerpo y su crecimiento espiritual que las lleve a comprender como relacionarse de una mejor manera con su entorno y lograr transacciones exitosas con sus familiares, vecinos amigos. Como lo manifiesta King (2000), lograr procesos interpersonales para conseguir una vida cotidiana óptima mediante continuos ajustes al estrés en los ambientes internos y externos, es un aporte importante para la vida de las mujeres hipertensas en su relación con su medio social, que les permite lograr las metas para el cuidado de su salud.

La interacción entre el personal de salud y la persona hipertensa exige empatía, confianza, comunicación efectiva, dialogo activo. El equipo de salud, debe aportar el reconocimiento de la persona enferma como ser individual, único y específico con sus características y condiciones particulares de las cuales se supedita la posible autonomía y capacidad de autocuidado (Holguín et al., 2006). La actitud y asistencia amable, empática y moralmente neutra, la pronta disponibilidad, la buena calidad de la comunicación y la interacción son atributos importantes de los profesionales de salud, determinantes para lograr una mayor adherencia de los pacientes a los tratamientos a largo plazo (Rodríguez y Gómez, 2010). Por su parte, la persona hipertensa debe ser miembro activo en la relación de cuidado, debe estar involucrado e informado; la información clara, suficiente, oportuna, ajustada a las necesidades, contribuye a una implicación activa del paciente en el tratamiento (Adams y Verna, 2004).

\section{CONCLUSIONES}

Las condiciones sociales en las cuales esta embebida la persona afectan las posibilidades de cumplimiento al tratamiento y del autocuidado, como son: tener bajo nivel de escolaridad, ser de bajo estrato socioeconómico, no pertenecer un sistema de seguridad e inestabilidad económica, son situaciones que afectan negativamente el cumplimiento al tratamiento, por las limitantes que representan para la comprensión y evolución de este fenómeno llamado hipertensión arterial.

El género femenino emerge como una condición limitante o un obstáculo en el autocuidado y seguimiento de las recomendaciones impartidas por la Institución de Salud, aparece ligado a lo anterior la expectativa sociocultural de "ser mujer": cuidadora por antonomasia, ser sumiso y solícito. Las características definidas por la etapa del ciclo vital, mujer en edad adulta, el ser madres cabeza de hogar fueron elementos que se agregaron a esa limitación. Ellas dieron prioridad o antepusieron la necesidad de garantizar la sostenibilidad económica de la familia, los cuidados del hogar, de los hijos y demás responsabilidades asignadas en su grupo familiar a sus propias necesidades de auto cuidado. Representa esto, una explicación al incumplimiento en el tratamiento y recomendaciones generales para la prevención de complicaciones y el cuidado de su salud.

Teniendo en cuenta que la hipertensión arterial sistémica es considerada el resultado de un proceso multifactorial, es necesario adelantar procesos de formación, motivación y seguimiento en las personas con diagnóstico de hipertensión arterial, en especial acerca de los factores modificables sobre los que puede 
actuar la persona enferma, sobre la importancia de modificar estos factores y de su participación activa en su recuperación, aun cuando no experimenten síntomas o alteraciones en su capacidad funcional, como en las fases iniciales de la enfermedad.

\section{REFERENCIAS}

- Adams, D; Verna, F; (2004) Su seguridad es importante para nosotros. Educación al paciente Jackson Health System. Disponible en: www.um-jmh.org/body. cfm=?id=8676 Consultado 10/03/2011

- Basaglia, F; Kanoussi, D; (1985) Mujer, Locura y Sociedad. Benemérita Universidad Autónoma de Puebla, México.

- Benner Associates. An Extended Statement on Narrative Accounts That Illustrate Nursing Practice (1991) [En línea],disponible en: http://home.earthlink. net/ bennerassoc/further.html.

- Bordignon, N.A. (2007) Desarrollo psicosocial de Erick Erickson. El diagrama epigenético del adulto. Rev. Lasallista de Investigación. 4(2), 7-16. Available from <http://www.scielo.org.co/scielo.php?script=sci_ arttext\&pid=S1794-44492007000200002\&lng=en\&nrm =iso $>$. access on 12 Dec. 2012

- Kasper D.L; Fauci, A.S; Longo, D.L; Braunwald, E; Hauser, S.L; Jameson, J.L. (2005) La práctica de la Medicina. En: Harrison. Principios de Medicina Interna. $16^{\mathrm{a}}$ edic. Mc Graw Hill, Barcelona, 1-6.

- Canaval, G. (2010) Salud de la mujer: Un desafío para el cuidado y un compromiso de todos. Editorial Universidad del Valle, Cali, 230

- Carvalho, A.S. (1991) Metodología da entrevista: Uma abordagem fenomenológica. Agir, Rio de Janeiro.

- Chobanian, A. et al. (2003) Hipertensión JNC. Seventh report of The Joint National.

- Committee on Prevention, Detection, Evaluation, And Treatment of High Blood Pressure. [En línea], disponible en: http://www.sld.cu/servicios/hta/doc/copiadeviijnc.pdf.
- Colunga, C. (2008) Algunos factores psicosociales en la hipertensión arterial. Universidad de Guadalajara: Departamento de ciencias médicas. [En línea], disponible en: http://www.tenlow.es/files/estrategia1.pdf

- Ministerio de la Protección Social. (2000) Programa de Apoyo a la reforma de Salud. Guía de atención de la Hipertensión Arterial. SA. . Ministerio de Salud de Colombia, Bogotá. Disponible en: http://www.nacer.udea. edu.co/pdf/libros/guiamps/guias13.pdf.

- Ministerio de la Protección Social (2000) Resolución 00412 de 2000: Guía de atención de la Hipertensión Arterial. Ministerio de Salud de Colombia, Bogotá.

- Durán de Villalobos, M.M. (2002) Marco epistemológico de la enfermería. Rev.Aquichan, Disponible en: http:// aquichan.unisabana.edu.co/index.php/aquichan/article/ view Article/17/34.

- Geertz, C.(1987) La Interpretación de las culturas. Gedisa, México.

- Holguín, L; Correa, D; Arrivillaga, M; et al. (2006) Adherencia al tratamiento de hipertensión arterial: efectividad de un programa de intervención biopsicosocial. Univ. Psychol. Bogotá 5 (3), 535-547. Disponible en: http://pepsic.bvs-psi.org.br/scielo.php?script=sci_ arttext\&pid=S1657-92672006000300009\&lng=es\& $\mathrm{nrm}=\mathrm{iso}>$. ISSN 1657-9267.

- Iza- Stoll, A. (2006) Tratamiento de la hipertensión arterial primaria. 23(2):93.

- Journal of the American College of Cardiology, Riesgo cardiovascular asociado con menopausia a temprana edad. Disponible en: http://www.encolombia.com/medicina/menopausia/Meno12206/Meno12206_Riesgo. html.

- Lagarde, M. (2004) Identidad femenina. Material didáctico de la División de estudios de postgrado de la Universidad Nacional Autónoma de México. Disponible en http://www.posgrado.unam.mx/servicios/productos

- Leininger, M; McFarland, M.R. (2002) Transcultural Nursing. Concepts, theories, research and practice. 3rd edn. McGraw-Hill, New York.

- National Heart Lung and blood Institute. People Science Health. Información para profesionales. Disponible en: http://www.nhlbi.nih.gov/guidelines/hypertension/ 
- Nigro. MaB; Herrera, H.L;, García, G; Raviolo, L; Ledesema, R.E. (2005) Evaluación de factores de riesgo cardiovascular en mujeres de la tercera edad Instituto del Corazón. Rev Rev Fed Arg Cardiol. (34), 358-365. Displonible en: http://www.fac.org.ar/1/revista/05v34n3/ art_orig/ar_ori01/nigro.PDF

- Organización Mundial de la Salud (2005) Una nueva publicación de la OMS analiza los factores de riesgo para la salud. Disponible en: http://www.who.int/mediacentre/ news/new/2005/nw04/es/

- Palma Campos, C. (2001) Mujeres con diabetes mellitus e hipertensión, la vivencia de su salud, y el autocuidado a partir de la construcción de la identidad femenina. Rev. cienc. adm. financ. segur. Soc 9(2). Disponible en: http://www.scielo.sa.cr/scielo.php?script=sci_ arttext\&pid=S1409-12592001000200009\&lng=es

- Polit, D; Hungler, B. (2000) Investigación científica en ciencias de la salud. Evaluación de datos cualitativos. Interamericana. McGraw-Hill, México.

- Ponty, M. (2008) La fenomenología existencial. Disponible en: http://alejandria.nidaval.com/scripts/Editorial. dll?SE=2_1_0_T0_A288_35
- Rengifo, J; Henríquez, E. (2010) Las pérdidas en el adulto mayor y su calidad de vida. Revista electrónica de portales médicos.com. 5(2),:24 Disponible en: http://www. portalesmedicos.com/publicaciones/articles/1985/1/ Las-perdidas-en-el-adultomayor-y-su-calidad-de-vida. html. Consultado 11/04/2011

- Rodríguez, A; Marín, D; (2007) Prevalencia de la hipertensión arterial sistólica aislada y factores de riesgo asociados en dos barrios del Municipio Independencia Estado Tachira. Rev haban cienc méd . Disponible en: http:// scielo.sld.cu/scielo.php?script=sci _arttext\&pid=S1729519X2007000200020\&lng=es.

- Rodríguez, A; Gómez, A. (2010) Factores influyentes en adherencia al tratamiento en pacientes con riesgo cardiovascular. Rev. Av en Enfermería. 28(1), 63-71.

- Rosano, M; Vitale, C; Tulli, A. (2007) Manejo del riesgo cardiovascular en mujeres menopáusicas,) Rev. Colombiana de Menopausia. 13(1), Disponible: http://www. encolombia.com/medicina/menopausia/Meno132007/ Manejo_riesgo_actualidad1.htm

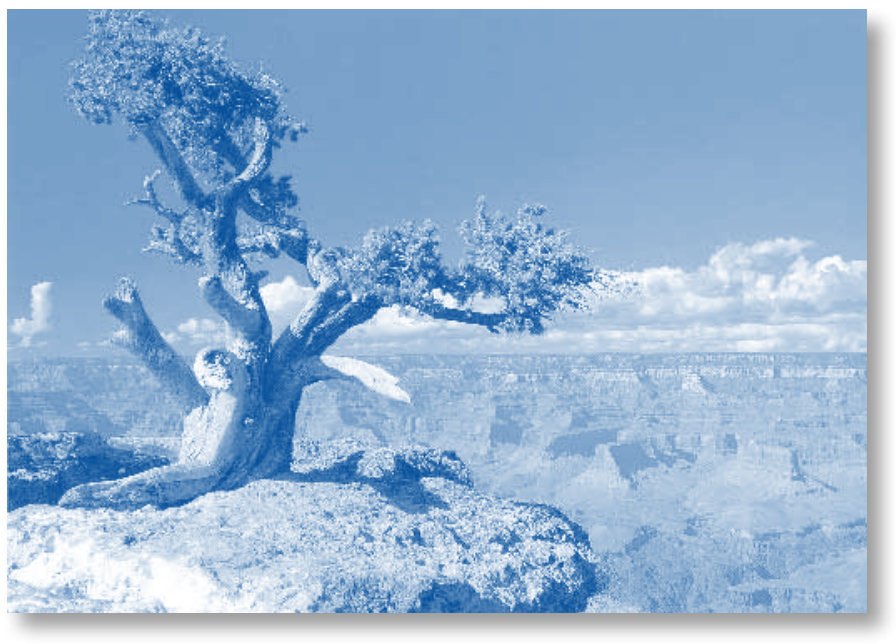

